\title{
Viral factors induce Hedgehog pathway activation in humans with viral hepatitis, cirrhosis, and hepatocellular carcinoma
}

Thiago de Almeida Pereira ${ }^{1,6}$, Rafal P Witek ${ }^{1}$, Wing-Kin Syn ${ }^{1}$, Steve S Choi ${ }^{1}$, Shelton Bradrick ${ }^{2}$, Gamze F Karaca ${ }^{1}$, Kolade M Agboola', Youngmi Jung ${ }^{1}$, Alessia Omenetti ${ }^{1}$, Cynthia A Moylan ${ }^{1}$, Liu Yang ${ }^{4}$, Martin E Fernandez-Zapico ${ }^{4,5}$, Ravi Jhaveri ${ }^{2,3}$, Vijay H Shah ${ }^{4}$, Fausto E Pereira ${ }^{6}$ and Anna M Diehl ${ }^{1}$

Hedgehog $(\mathrm{Hh})$ pathway activation promotes many processes that occur during fibrogenic liver repair. Whether the Hh pathway modulates the outcomes of virally mediated liver injury has never been examined. Gene-profiling studies of human hepatocellular carcinomas (HCCs) demonstrate Hh pathway activation in HCCs related to chronic infection with hepatitis B virus (HBV) or hepatitis C virus (HCV). Because most HCCs develop in cirrhotic livers, we hypothesized that $\mathrm{Hh}$ pathway activation occurs during fibrogenic repair of liver damage due to chronic viral hepatitis, and that Hh-responsive cells mediate disease progression and hepatocarciongenesis in chronic viral hepatitis. Immunohistochemistry and qRT-PCR analysis were used to analyze Hh pathway activation and identify Hh-responsive cell types in liver biopsies from 45 patients with chronic HBV or HCV. Hh signaling was then manipulated in cultured liver cells to directly assess the impact of $\mathrm{Hh}$ activity in relevant cell types. We found increased hepatic expression of $\mathrm{Hh}$ ligands in all patients with chronic viral hepatitis, and demonstrated that infection with HCV stimulated cultured hepatocytes to produce Hh ligands. The major cell populations that expanded during cirrhosis and HCC (ie, liver myofibroblasts, activated endothelial cells, and progenitors expressing markers of tumor stem/initiating cells) were Hh responsive, and higher levels of Hh pathway activity associated with cirrhosis and HCC. Inhibiting pathway activity in Hh-responsive target cells reduced fibrogenesis, angiogenesis, and growth. In conclusion, $\mathrm{HBV} / \mathrm{HCV}$ infection increases hepatocyte production of $\mathrm{Hh}$ ligands and expands the types of Hh-responsive cells that promote liver fibrosis and cancer.

Laboratory Investigation (2010) 90, 1690-1703; doi:10.1038/labinvest.2010.147; published online 9 August 2010

KEYWORDS: fibrosis; Hedgehog pathway; hepatitis B; hepatitis C; liver progenitors; morphogens

Cirrhosis and liver cancer are major causes of morbidity and mortality. Worldwide, most cases of cirrhosis and liver cancer are caused by chronic infection with hepatitis B (HBV) or C viruses (HCV). ${ }^{1,2}$ Unfortunately, despite effective vaccination for hepatitis $\mathrm{B}$ and improvements in antiviral therapies for both hepatitis $\mathrm{B}$ and hepatitis $\mathrm{C}$, the global burden of chronic hepatitis B and/or hepatitis $C$ infection and virally mediated liver disease remains enormous. ${ }^{2,3}$

Progressive liver damage from chronic HBV/HCV occurs because chronic viral infection increases the death rate of hepatocytes. ${ }^{4}$ This triggers repair responses to replace the cells that died. ${ }^{4}$ In some individuals, however, repair is fibrogenic and results in progressive accumulation of collagen matrix. ${ }^{5}$ Fibrogenic repair is also generally accompanied by vascular remodeling and hepatic accumulation of liver progenitor cells. ${ }^{5}$ Expansion of liver progenitor populations, in turn, provides a source of immature cells with survival advantages, some of which may eventually become tumorinitiating cells for primary liver cancers. ${ }^{6}$ Primary hepatocellular carcinoma (HCC) is a major cause of liverrelated death in HBV/HCV-infected individuals with cirrhosis. ${ }^{3,4}$ Therefore, fibrogenic repair contributes to the

\footnotetext{
'Division of Gastroenterology, Duke University, Durham, NC, USA; ${ }^{2}$ Department of Molecular Genetics and Microbiology, Duke University, Durham, NC, USA; ${ }^{3}$ Department of Pediatrics, Duke University, Durham, NC, USA; ${ }^{4}$ Division of Gastroenterology and Hepatology, Division of Oncology Research, Mayo Clinic, Rochester, MN, USA; ${ }^{5}$ Schulze Center for Novel Therapeutics, Division of Oncology Research, Mayo Clinic, Rochester, MN, USA and ${ }^{6}$ Núcleo de Doenças Infecciosas, Centro de Ciências da Saúde, Universidade Federal do Espírito Santo, Vitória, ES, Brazil

Correspondence: Dr AM Diehl, MD, Florence McAlister Professor and Chief, Division of Gastroenterology, Duke University, Snyderman Building (GSRB-1), 595 LaSalle Street, Suite 1073, Durham, NC 27710, USA.

E-mail: diehl004@mc.duke.edu

Received 10 May 2010; revised 6 July 2010; accepted 7 July 2010
} 
pathogenesis of the potentially fatal outcomes of chronic viral hepatitis.

Recent studies of cultured cells, animal models of metabolic and biliary types of liver disease, and small numbers of liver biopsy samples from patients with similar conditions have identified a mechanism that modulates fibrogenic repair, namely, activation of the Hedgehog ( $\mathrm{Hh}$ ) signaling pathway. ${ }^{7-9}$ Hh ligands are morphogens that orchestrate tissue construction and remodeling by initiating autocrine and paracrine signaling in Hh-responsive cells. ${ }^{10}$ In such cells, interaction of Hh ligands with Patched (Ptc) receptors on the cell surface releases the signaling-competent co-receptor, Smoothened (Smo), from Ptc repression. This permits intracellular signal transduction that culminates in the nuclear localization of Glioma-associated oncogene family transcription factors (Gli1, 2, and 3) that regulate the transcription of Hh-responsive genes. ${ }^{10}$ Hepatic stellate cells (HSCs), some cholangiocytes, liver progenitors, and lymphocytes are capable of producing Hh ligands. ${ }^{8,11-13}$ Hh ligands function as viability factors for multipotent progenitors that are capable of differentiating into mature hepatocytes or cholangiocytes. ${ }^{11}$ They also stimulate quiescent HSCs to acquire a more myofibroblastic phenotype, and promote the proliferation and survival of myofibroblastic cells. ${ }^{13}$ In addition, Hh ligands act in a paracrine fashion to induce cultured ductular cells to undergo epithelial-to-mesenchymal transition (EMT), providing another mechanism that might increase myofibroblastic cell numbers in livers. ${ }^{8,14,15}$ In vitro, myofibroblast-derived Hh ligands upregulate expression of various chemokines and chemokine receptors in neighboring immature liver epithelial cells, facilitating hepatic recruitment and retention of certain types of immune cells. ${ }^{16}$ Some of these immune cells, in turn, produce and respond to Hh ligands, ${ }^{12,16}$ further enriching the microenvironment with morphogens. Finally, Hh ligands may influence vascular remodeling during liver damage because they exert pro-angiogenic effects during development and adult wound healing responses. ${ }^{17,18}$ Indeed, liver sinusoidal endothelial cells (SECs) were recently shown to be Hh-responsive cells that upregulate their expression of adhesion molecules and other markers of activated sinusoidal endothelium when exposed to Hh ligands. ${ }^{19}$ Hence, there is growing experimental evidence that $\mathrm{Hh}$ signaling increases during liver injury and that activation of the Hh pathway promotes many processes that are known to occur during fibrogenic liver repair, including growth of myofibroblastic populations, EMT, vascular remodeling, and hepatic accumulation of inflammatory cells and liver progenitors.

Whether or not the Hh pathway modulates the outcomes of virally mediated liver injury has never been examined. Recent gene-profiling studies of human liver cancer repositories provide evidence for Hh pathway activation in a substantial proportion of $\mathrm{HBV} / \mathrm{HCV}$-related $\mathrm{HCCs}^{20-22}$ Given that virally induced cirrhosis is a strong risk factor for HCC, the latter data suggest that Hh pathway activation might occur during chronic viral hepatitis. If verified, this raises additional questions about the role(s) of Hh-responsive cells in regulating disease progression and hepatocarcinogenesis during virally mediated liver disease. The goals of this study, therefore, were to determine if the Hh pathway becomes activated in the livers of patients with chronic viral hepatitis; to characterize the types of liver cells that produce and/or respond to Hh ligands in virus-infected livers; and to delineate potential mechanisms by which $\mathrm{Hh}$ signaling might contribute to fibrotic repair and hepatocarcinogenesis in this context. We hypothesized that $\mathrm{Hh}$ signaling would increase in cell populations that mediate fibrogenic repair during chronic viral hepatitis and induce such cells to acquire phenotypes that promote liver fibrosis, vascular remodeling, and hepatocarcinogenesis. Hence, fibrosis stage and HCC development were predicted to increase in parallel with the level of Hh pathway activity in patients with chronic viral hepatitis.

\section{MATERIALS AND METHODS Human Samples}

A total of 45 formalin-fixed paraffin-embedded (FFPE) liver biopsies from subjects with progressive hepatitis $\mathrm{B}(n=23)$ and C $(n=22)$ were obtained from the Department of Pathology tissue repository at Universidade Federal do Espírito Santo, Brazil (see details in Supplementary Materials and Methods). The demographic data of selected subjects are described in Table 1. FFPE liver sections were also obtained from residual healthy liver tissues of two donor livers that were utilized for split liver transplantation at Duke University Hospital. The study was approved by the human ethics committee of Centro de Ciências da Saúde, Universidade Federal do Espírito Santo (033-09) and by the Duke institutional review board, and it fulfilled Institutional and NIH Guidelines for human subject research.

\section{Histology and Immunohistochemistry (IHC)}

FFPE livers were prepared for IHC and serial sections were stained with H\&E and Sirius red. For each immunostain, sections from control livers and sections from livers with viral hepatitis were always processed identically and concurrently. Each assay also always included appropriate positive controls (other types of diseased human liver with known Hh pathway activation) and negative controls (diseased human liver incubated with secondary antibody but without primary antibody). Detailed protocols are in Supplementary Materials and Methods and antibodies used are listed in Supplementary Table 1.

\section{Cell Lines and Studies to Assess Effects of Viral Proteins on Hh Ligand Production}

Huh-7 cells (gift from Eckard Wimmer, Stony Brook University, NY, USA) were grown according to standard protocols. $^{23}$ After pilot experiments were completed to establish optimal conditions for viral infection, Huh-7 cells were infected with HCV genotype 2a JFH-1 virus (MOI of 0.1$)^{24,25}$ or control adenoviral vectors (gift from P Goldschmidt-Clermont, University of Miami, FL, USA; MOI of 50$)^{26}$ and harvested $72 \mathrm{~h}$ later for RNA isolation/analysis. 
Table 1 The demographic data of the selected patients from whom the tissue was used for the study

\begin{tabular}{|c|c|c|c|c|c|c|c|}
\hline \multicolumn{8}{|l|}{$H B V$} \\
\hline F1 & 5 & $31.6( \pm 9)$ & $4 \mathrm{M} / 1 \mathrm{~F}$ & $0 / 1 / 2$ & Pos & $40.6( \pm 12)$ & $36.7( \pm 10)$ \\
\hline $\mathrm{F} 2$ & 4 & $41.8( \pm 10)$ & $3 \mathrm{M} / 1 \mathrm{~F}$ & $2 / 2 / 2$ & Pos & $56.7( \pm 3)$ & $50.0( \pm 10)$ \\
\hline & No. of patients & Average age & Gender & Inflammation & Genotype & ALT & AST \\
\hline \multicolumn{8}{|l|}{$\mathrm{HCV}$} \\
\hline F0 & 4 & $47.3( \pm 15)$ & $3 \mathrm{M} / 1 \mathrm{~F}$ & $0 / 1 / 1$ & 1.3 & $55.0( \pm 24)$ & $41.0( \pm 17)$ \\
\hline & No. of patients & Average age & Gender & ALT & AST & & \\
\hline \multicolumn{8}{|l|}{$\mathrm{HCC}$} \\
\hline HBV & 4 & $66.2( \pm 12)$ & $3 \mathrm{M} / 1 \mathrm{~F}$ & $35.7( \pm 26)$ & $28.6( \pm 14)$ & & \\
\hline $\mathrm{HCV}$ & 3 & $57.3( \pm 15)$ & $3 M$ & $117.5( \pm 40)$ & $90.5( \pm 46)$ & & \\
\hline
\end{tabular}

Isolation and Culture of Human Primary HSCs and SECs to Assess Effects of Hh Pathway Activity

Approximately $25 \mathrm{~g}$ of finely cut liver obtained from a residual segment of healthy human liver was used for primary isolation of HSCs and SECs as previously described. ${ }^{27,28}$

To assess the effects of Hh pathway activity, primary HSCs and SECs were treated with cyclopamine $(5 \mu \mathrm{M}$, Toronto Research Chemicals, Toronto, Canada), an inhibitor of $\mathrm{Hh}$ signaling, or its catalytically inactive analog, tomatidine $(5 \mu \mathrm{M}) \cdot{ }^{29} \mathrm{RNA}$ and protein were harvested for qRT-PCR and western blot analysis $48 \mathrm{~h}$ later.

\section{Angiogenesis Assay}

A total of 12000 primary human SECs (from ScienceCell, San Diego, CA, USA) were seeded in each well of chamber slide with $100 \mu$ l Matrigel after $30 \mathrm{~min}$ of reincubation at $37^{\circ} \mathrm{C} .^{30}$ Wells were photographed at different time points at random fields with the use of a confocal microscope (Zeiss LSM Pascal Axiovert; Carl Zeiss, Welwyn Garden City, Hertfordshire, UK). The length of the vascular tubes after $16 \mathrm{~h}$ was digitally analyzed using AnalySISD software (Olympus Biosystems, Münster, Germany). Cultures were treated with vehicle, recombinant sonic hedgehog (Shh; $5 \mu \mathrm{g} / \mathrm{ml}$; Stem Cell Tech, Vancouver, Canada), primary HSC (from ScienceCell) conditioned medium with or without cyclopamine, or pre-treated with short hairpin RNAs (shRNAs) targeted against Smo to determine effects on tube formation, a wellcharacterized surrogate for angiogenesis. ${ }^{30}$ shRNA was designed as previously described; ${ }^{31}$ Smo shRNA sequence is shown in
Supplementary Table 2. For the shRNA delivery we used the BLOCK-iT Inducible H1 Lentiviral RNAi System (Invitrogen).

\section{RNA and Protein Analysis}

Real-time reverse transcription-polymerase chain reaction (RT-PCR) and western immunoblot were performed using established protocols; ${ }^{12}$ the details are in Supplementary Materials and Methods and Supplementary Table 3.

\section{RNA Isolation from FFPE Tissue and qRT-PCR}

Total RNA was isolated from archival FFPE tissue using RNeasy FFPE kit (Qiagen, Valencia, CA, USA) following the manufacturer's protocols. As the concentration of obtained cDNA was very low, qRT-PCR cycles were increased to 50 (60 for Laser Capture samples), and all work involving mixing of reagents and loading of plates was performed in a sterile hood to prevent contamination.

For laser capture microdissection (LCM) Palm MicroBeam Microlaser and Zeiss microscope were used. First, FFPE tissue was deparaffinized in xylene for $5 \mathrm{~min}$ and was then cut using Auto-LPC option that allows catapulting of selected tissue parts into collection vessel. Following tissue collection, RNA was isolated using RNeasy FFPE kit. Final cDNA was diluted to $50 \mu \mathrm{l}$, and $5 \mu \mathrm{l}$ was used per reaction.

\section{Statistical Analysis}

Results are expressed as means \pm s.e.m. Comparisons between groups were performed using the nonparametric Wilcoxon 
rank-sum test using SAS version 9.1 software (SAS Institute, Cary, NC, USA). The $P$-values are two tailed; significance was accepted at the $5 \%$ level.

\section{RESULTS}

\section{Patient Populations}

In all, 22 patients with liver disease caused by chronic HBV infection and 23 patients with chronic HCV-induced liver disease underwent clinically indicated liver biopsies, and residual, de-identified FFPE tissues were used for this study. Table 1 summarizes the clinical information that was submitted with each liver biopsy. Males predominated in the subgroups with HBV and HCC. Patients tended to be middle aged. However, the group without cancer was generally about one decade younger than the group with coincident HCC. Serum aminotransferase values were less than two-fold elevated in patients with chronic HBV, including the subgroups with advanced fibrosis and/or HCC. HCV patients who had no fibrosis (F0) or only mild-moderate fibrosis (F1/F2) also had relatively normal serum aminotransferases, whereas HCV patients with F3/F4 fibrosis and/or HCC had significant aminotransferase elevations.

\section{Chronic Viral Hepatitis Increases Hepatic Production of Hh Ligands}

Compared with liver biopsies from controls without chronic liver disease that rarely, if ever, demonstrated cells that expressed Hh ligands (Figure 1a, Supplementary Figure 1A, and Supplementary Figure 2A and B), biopsies from patients with viral hepatitis demonstrated significantly increased expression of Shh and Indian hedgehog (Ihh) mRNAs, as well as accumulation of cells that produced these proteins (Figure $1 \mathrm{~b}$ and $\mathrm{c}$ and Supplementary Figure $1 \mathrm{~B}$ and $\mathrm{C}$ ). Increased expression of Shh and Ihh mRNAs was noted consistently in virus-infected patients with relatively little liver fibrosis, but higher levels of Shh and Ihh mRNAs were generally exhibited by patients with bridging fibrosis. In patients with HBV, mRNA levels of Shh and Ihh remained relatively flat across the spectrum of more advanced fibrosis, whereas levels of ligand mRNAs more closely paralleled fibrosis stage in $\mathrm{HCV}$ (Figure $1 \mathrm{~b}$ and Supplementary Figure 1B). Shh protein displayed a strong positive correlation with fibrosis stage in HBV and HCV (Supplementary Figure 2C and D), but not with inflammatory grade (data not shown). In both diseases, the types of cells that were producing Hh ligands differed according to fibrosis stage. In livers with relatively little fibrosis (F0/F1), production of Shh occurred predominately in hepatocytes, although the ductular cells that were present also expressed this ligand. In contrast, Ihh production tended to occur mainly in stromal and sinusoidal cells in livers with F0/F1 fibrosis. As fibrosis advanced, the ductular reaction increased, Shh production progressively shifted to stromaland ductular-type cells, and these cell types were the predominant source of Shh in livers with advanced fibrosis (F3/F4), although Shh production remained detectable in some hepatocytes (Figure 1c and Supplementary Figure 3A and D). During advanced fibrosis, however, Ihh expression remained largely confined to the stromal compartment (Supplementary Figures $1 \mathrm{C}$ and $3 \mathrm{E}$ and F).

Evidence for substantially increased production of $\mathrm{Hh}$ ligands in early-stage viral hepatitis suggested that viral infection per se might induce expression of Shh and/or Ihh mRNAs in liver cells. We infected cultured human hepatoma cells (Huh-7) with HCV (JFH-1 strain) to address this issue. Cell culture-derived HCV JFH-1 virus was incubated with Huh-7 cells and Hh ligand expression was assessed by qRTPCR $72 \mathrm{~h}$ later. Results were compared with Shh expression in Huh-7 cells that had been infected with various adenoviral vectors. Although none of the adenovirus-infected cells demonstrated increases in Shh mRNA levels (data not shown), Shh mRNA expression was significantly induced in cells that were infected with HCV JFH-1 virus (Figure 1d). These findings complement the IHC data and suggest that infection of hepatocytes with the hepatitis virus directly stimulates such cells to produce Shh. Also, consistent with the IHC evidence that stromal cells (rather than liver epithelial cells) were the major producers of Ihh during chronic viral infections, viral factors did not influence expression of Ihh in the cultured liver cells (data not shown).

\section{Accumulation of Hh-Responsive Cells Correlates with Fibrosis Stage in Viral Hepatitis}

We next determined if production of Hh ligands resulted in expression of Hh-regulated genes, such as Ptc and Gli2. Healthy control livers harbored only rare Gli2 $(+)$ or $\operatorname{Ptc}(+)$ cells, and these localized within and near portal tracts (Figure 2a and Supplementary Figure 4A). In contrast, livers that were infected with $\mathrm{HBV}$ or $\mathrm{HCV}$ demonstrated significantly increased mRNA levels of Ptc and Gli2, with greatest expression of both target gene mRNAs occurring in livers with bridging fibrosis (Figure 2b and Supplementary Figure 4B). IHC verified that cells expressing Ptc and Gli2 proteins accompanied the accumulation of Ptc and Gli2 mRNAs, and revealed that the distribution of such Hhresponsive cells varied with fibrosis stage (Figure $2 \mathrm{c}$ and Supplementary Figure 4C and D). In livers with relatively little fibrosis (F0/F1), Ptc $(+)$ cells were scattered throughout the lobular parenchyma where they mainly localized along sinusoids. Gli2 $(+)$ cells were localized mainly in and around portal tracts in livers with F0/F1 fibrosis. In livers with advanced (F3/F4) fibrosis, cells that expressed Ptc and/or Gli2 appeared to localize mainly within/along fibrous septae, although the numbers of Hh-responsive sinusoidal cells within the lobular parenchyma also increased.

\section{Hh-Responsive Cells Localize in Areas of Fibrosis and Liver Myofibroblasts Require Hh Pathway Activity to Retain Their Fibroblastic Phenotype}

To better characterize populations of Hh-responsive cells, serial sections were stained with Sirius red to demonstrate 
fibrosis and $\alpha$-SMA to demonstrate myofibroblasts (Supplementary Figure 5). As expected, Sirius red staining increased with fibrosis stage in both HBV and HCV (Supplementary Figure 5A). Consistent with evidence that myofibroblasts are
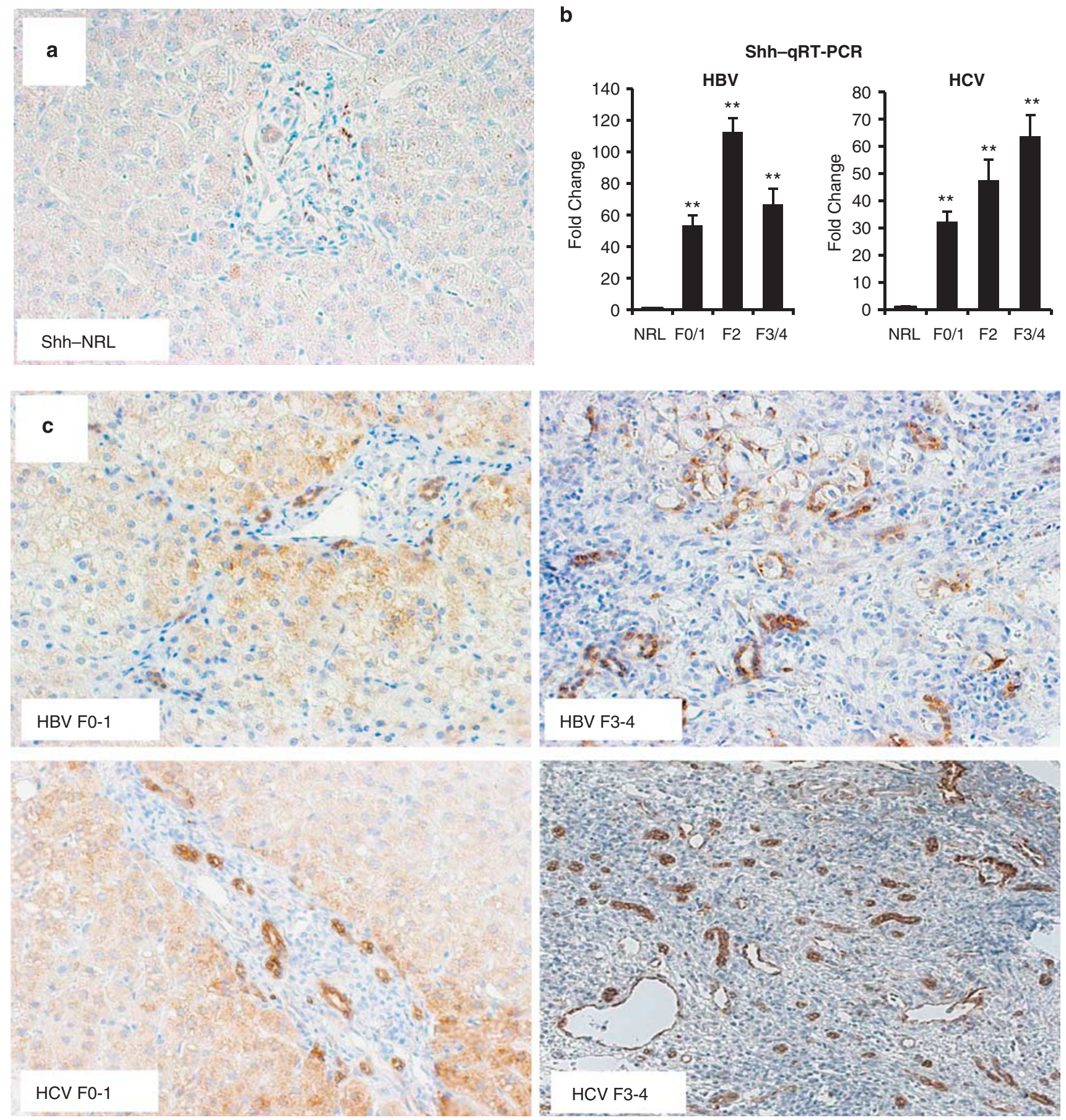

Figure 1 Liver cell production of sonic Hh ligands (Shh) increases during chronic viral hepatitis. (a) Liver section from a representative control liver without viral hepatitis (NRL). Final magnification $\times 200$. (b) qRT-PCR analysis of RNA isolated from normal liver, and HBV- or HCV-infected livers with F0/F1, F2, or F3/F4 fibrosis ( $n=3$ per group). Results were normalized to Shh expression in normal control livers; means \pm s.e.m. are displayed. ${ }^{* * P<0.005 ~ v s ~ c o n t r o l s . ~}$ (c) Immunohistochemical staining for Shh in representative sections from patients with HBV (top) and HCV (bottom). Left panels display findings in livers with F0 fibrosis; right panels display findings in livers with F3/F4 fibrosis. Final magnification $\times 200$. (d) qRT-PCR analysis of Shh expression in Huh-7 cells infected with HCV JFH-1. Results were normalized to the respective controls and mean \pm s.e.m. data are shown. ${ }^{* * P}<0.005$ vs controls. 


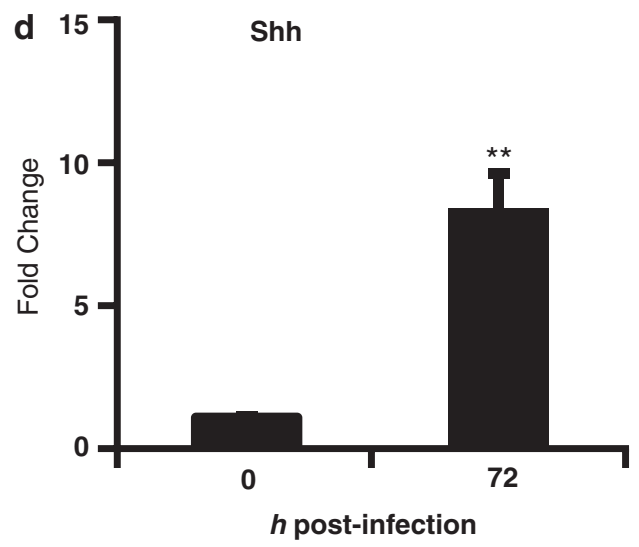

Figure 1 (continued)

immunostained sections at higher magnification demonstrated that sinusoids harbored many $\alpha$-SMA $(+)$ cells, even in livers with relatively little fibrosis (stage F0/F1); cells that produced Ihh and Hh-responsive (ie, Ptc-positive) cells were similarly distributed (Figure $3 \mathrm{~b}$ ). These findings suggested that the myofibroblast population was enriched with Hh-responsive cells.

To more directly examine the potential significance of $\mathrm{Hh}$ signaling in myofibroblastic liver cells, HSCs were isolated from residual healthy liver allograft tissue, and cultured to induce myofibroblastic transdifferentiation. Myofibroblastic human HSCs were then treated with cyclopamine, a highly specific Hh signaling antagonist, or its biologically inactive analog, tomatidine. Primary myofibroblastic HSCs that were treated with tomatidine demonstrated $\mathrm{Hh}$ pathway activation, as evidenced by expression of Hh target genes (Gli2), as well as the expected production of various myofibroblast markers, such as $\alpha$-sma and collagen $1 \alpha 1$ (Coll $\alpha 1$; Figure 3c and d). In contrast, treating myofibroblastic HSCs with cyclopamine inhibited $\mathrm{Hh}$ signaling and significantly repressed expression of myofibroblast-related genes (Figure $3 \mathrm{c}$ and $\mathrm{d}$ ). These in vitro data complement the findings in whole liver tissue, and suggest that the Hh pathway has an important role in maintaining the myofibroblastic phenotype of stromal cells that accumulate during the progression of chronic viral hepatitis.

\section{Hh Ligands Increase Expression of Activation Markers in Human Hepatic SECs and Promote Angiogenesis}

Further inspection of the fibrous septae in immunostained sections of HBV- or HCV-infected livers with advanced fibrosis demonstrated Ptc-expressing cells and cells with Gli2positive nuclei lining apparent vascular spaces (Supplementary Figure 6A and B). Strong expression of CD31, a marker of activated SECs, was also noted in these areas (Supplementary Figure 6C). In order to determine if the activated endothelial cells were $\mathrm{Hh}$ responsive, additional sections were double stained for CD31 and Gli2. Many cells that lined vascular spaces within fibrous septae coexpressed CD31 and Gli2 in livers with chronic viral hepatitis, indicating that activated hepatic endothelial cells are $\mathrm{Hh}$ responsive (Figure 4a).

To more directly assess the role of Hh signaling in hepatic vascular remodeling, primary endothelial cells were isolated from residual human liver allograft tissue from a healthy donor, placed in culture to induce activation, and then treated with cyclopamine or tomatidine. Culture-activated endothelial cells exhibited evidence of Hh pathway activation (increase in Gli2 mRNA level); cyclopamine treatment inhibited Hh signaling and repressed endothelial cell gene expression of the activation marker CD31 (Figure 4b). In separate experiments, human SECs were cultured in matrigel and Hh signaling was manipulated to determine effects on vascular tube formation. In this angiogenesis model, treatment with recombinant Shh or myofibroblast-conditioned medium (a source of Hh ligands) stimulated tube formation, whereas various strategies that inhibited $\mathrm{Hh}$ signaling blocked tube formation (Figure $4 \mathrm{c}-\mathrm{e}$ ). These in vitro data support the concept that Hh ligands released from liver cells provide paracrine signals that lead to Hh pathway activation in neighboring endothelial cells, thereby regulating vascular remodeling responses that occur in livers with chronic viral hepatitis.

\section{Hh-Responsive Cells in the Ductular Reaction Coexpress Markers of Liver Epithelial Progenitors and Mesenchymal Cells}

Virally infected livers with advanced fibrosis demonstrated increased numbers of ductular structures embedded within fibrous septae (Figures 1c, 2c, and 5, and Supplementary Figure 4C and D). Many of the cells in such ductular structures stained positively for Shh (Figures $1 \mathrm{c}$ and $5 \mathrm{a}$, and Supplementary Figure 3C and D) and some had Gli2(+) nuclei (Figures $2 \mathrm{c}$ and 5b, and Supplementary Figure 7), demonstrating that many epithelial cells in the ductular reaction were producing and responding to Hh ligands. Autocrine $\mathrm{Hh}$ signaling is a general property of progenitor cells. $^{11,32}$ Therefore, we used IHC to assess progenitor markers in the ductular cells of the virally infected livers. The results indicate that such cells generally express several wellaccepted markers of liver epithelial progenitors, including Krt7 (Figure 5c) and CD133 (Figure 5d). Interestingly, many of these cells also stained positively for typical mesenchymal markers, such as $\alpha$-SMA (Figure 5e) and S100A4 (Figure 5f). Coexpression of epithelial and mesenchymal genes is an accepted characteristic of cells that are undergoing EMT. ${ }^{14,33}$ Thus, the new results support earlier work that showed that Hh pathway activation induced EMT in immature ductular cells, ${ }^{8}$ and demonstrate that livers with chronic viral hepatitis become enriched with populations of Hh-responsive progenitors that are undergoing EMT. 


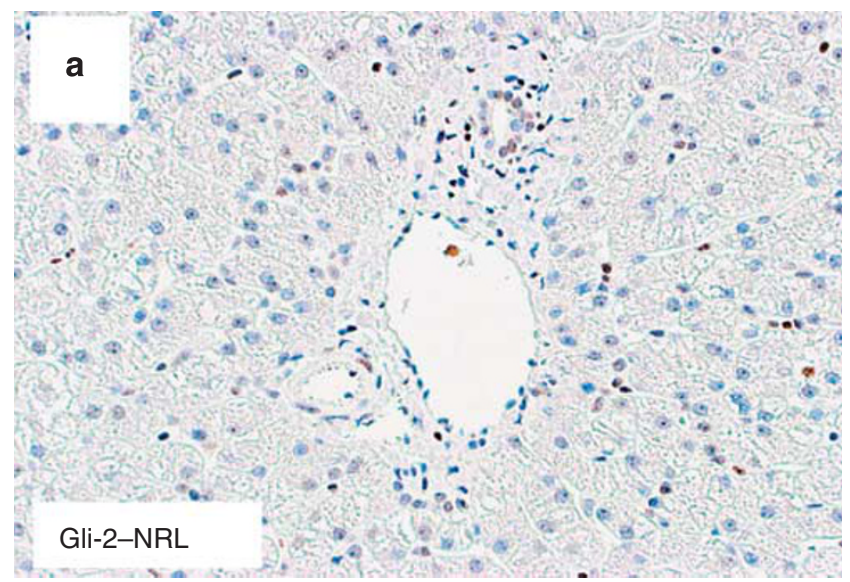

b
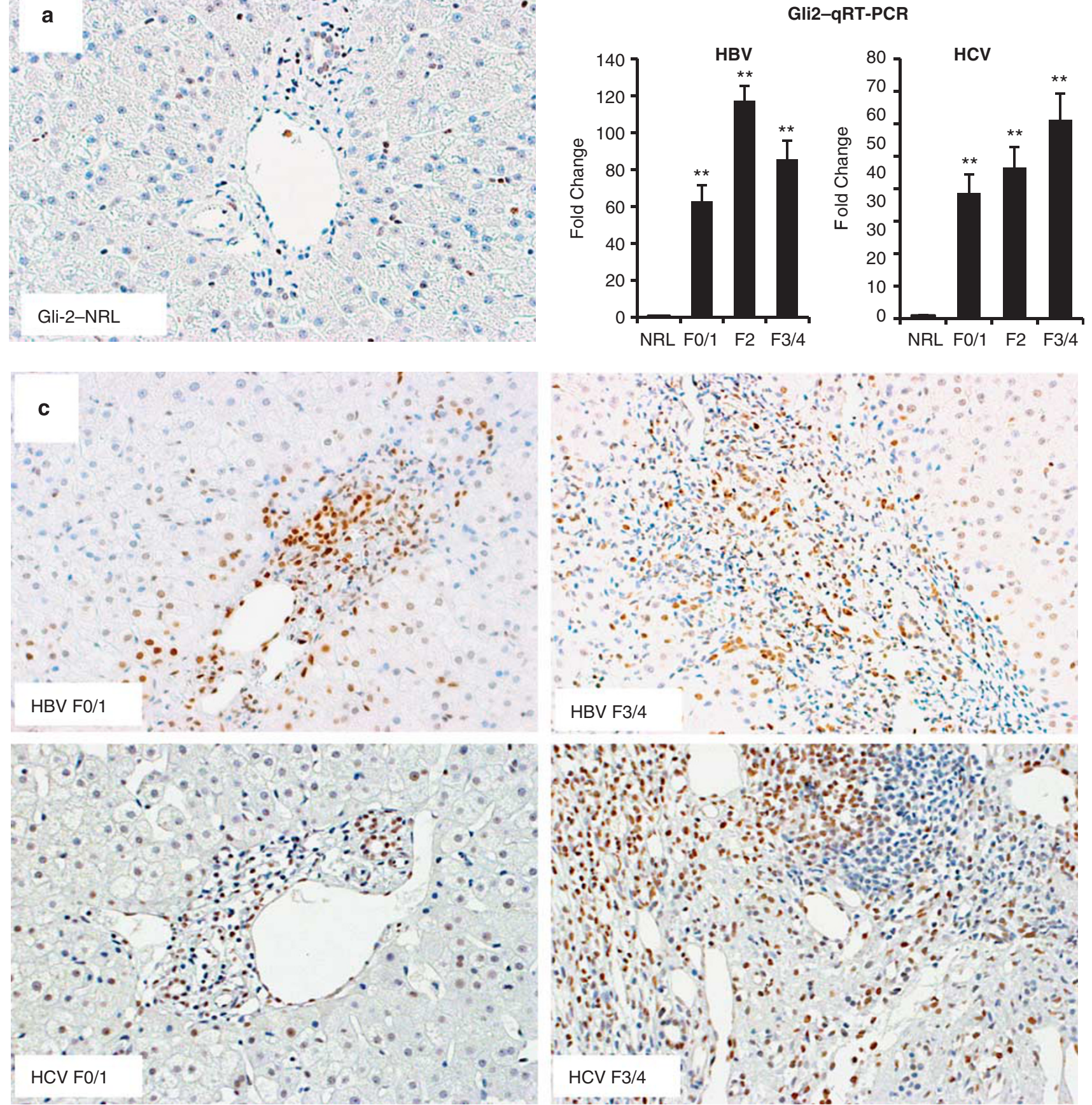

Figure 2 Liver cells that express the Hh-regulated transcription factor, Gli2, accumulate during chronic viral hepatitis. (a) Liver section from a representative control liver without viral hepatitis (NRL). Final magnification $\times 200$. (b) qRT-PCR analysis of RNA isolated from normal liver, and HBV- or HCV-infected livers with F0/F1, F2, or F3/F4 fibrosis ( $n=3$ per group). Results were normalized to Gli2 expression in normal control livers; mean \pm s.e.m. data are displayed. ${ }^{*}{ }^{*}<0.005$ vs controls. (c) Immunohistochemical staining for Gli2 in representative sections from patients with HBV (top) and HCV (bottom). Left panels display findings in livers with F0 fibrosis; right panels display findings in livers with F3/F4 fibrosis. Final magnification $\times 200$.

\section{Hh-Responsive Cells Are Particularly Enriched in Peritumoral Stroma of HCCs}

Because chronic viral hepatitis is a major risk factor for HCC, ${ }^{2,3}$ and Hh pathway activation has been demonstrated in certain HCC cell lines, ${ }^{21}$ we used IHC to localize $\mathrm{Hh}$ ligand-producing cells and Hh-responsive cells in livers with chronic viral hepatitis and co-incident HCC. To our surprise, we were generally unable to demonstrate Shh, Ihh, Ptc, or Gli2 staining in malignant hepatocytes. However, the peritumoral stromal tissue was enriched with cells that expressed Ptc and S100A4. LCM and QRT-PCR analysis revealed robust expression of Ptc mRNA in the fibrous tissue that 

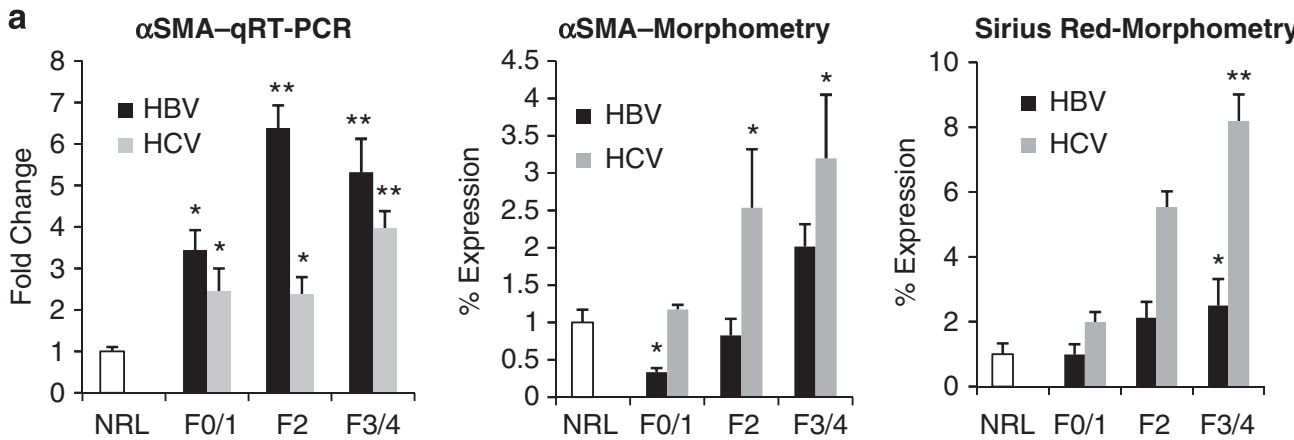

b
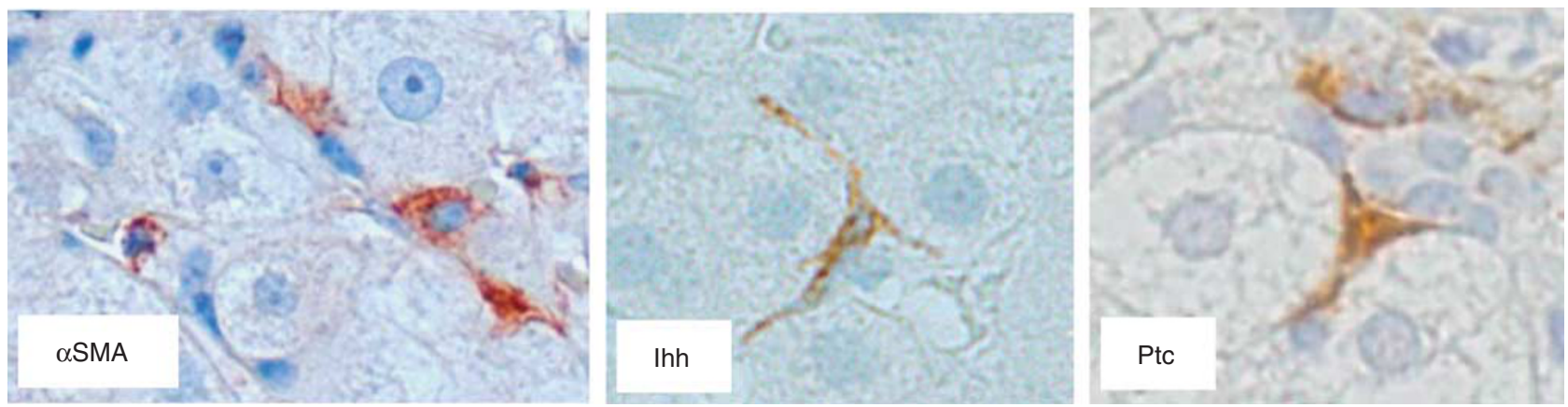

C

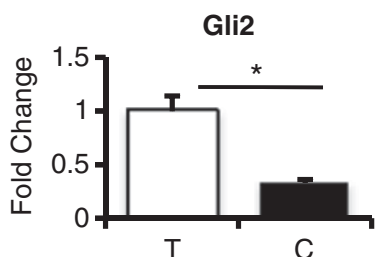

Primary Human HSC

$\alpha$ SMA

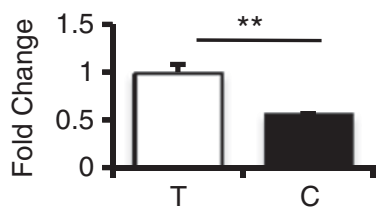

Col1 $\alpha 1$

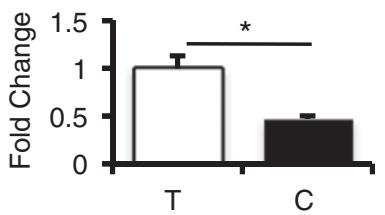

d

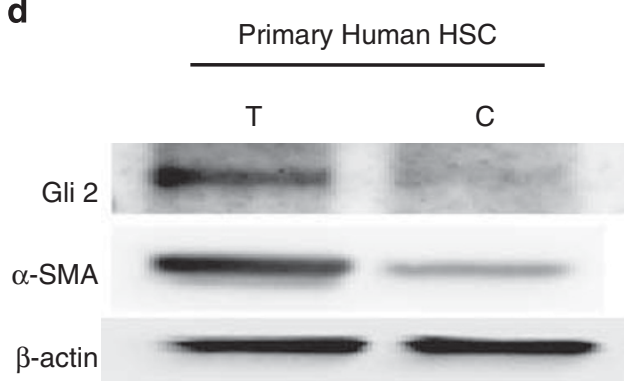

Figure 3 Myofibroblastic cells that accumulate during chronic viral hepatitis are $\mathrm{Hh}$ responsive, and Hh signaling is necessary for fibrogenic gene expression. (a) RNA was isolated from normal liver, and HBV- or HCV-infected livers with F0/F1, F2, or F3/F4 fibrosis ( $n=3$ per group); qRT-PCR was done to assess expression of $\alpha$-sma (left panel); accumulation of $\alpha$-SMA-expressing cells (middle panel) and Sirius red-stained fibrils (right panel) were quantified in serial liver sections using morphometric analysis. Means \pm s.e.m. results are graphed. ${ }^{\star} P<0.05,{ }^{*} P<0.005$ vs controls. (b) Immunohistochemistry demonstrating sinusoidal cell expression of $\alpha$-SMA (left panel), Ihh (middle panel), and Patched (Ptc) (right panel) in representative liver sections with F0/F1 fibrosis. Final magnification $\times 600$. (c, d) Hepatic stellate cells were isolated from non-diseased human liver and cultured to induce transition to myofibroblastic cells (MF); then treated with tomatidine (T) or cyclopamine (Cy) for an additional $48 \mathrm{~h}$. RNA and protein were isolated from triplicate plates and qRT-PCR (c) and western blot (d) were used to assess effects on gene expression. Experiments were replicated a total of three times; data were normalized to tomatidine-treated cultures. Means \pm s.e.m. are displayed. ${ }^{*} P<0.05,{ }^{* *} P<0.005$ vs tomatidine-treated cultures.

surrounded tumor nodules (Figure 6). Closer inspection revealed that this fibrous tissue was riddled with vascular spaces and enriched with poorly formed ductular structures. IHC demonstrated that fibrous tissue adjacent to tumors harbored numerous ductular structures that expressed Shh and Gli2, as well as stromal cells that expressed Ihh, Gli2 (Figure 7a and c) and/or Ptc (Supplementary Figure 7B).
Further immunostaining demonstrated that these ductular structures tended to express Krt7, a marker of immature liver epithelial cells (Figure 7d and Supplementary Figure 7C); most also stained positively for the tumor stem/progenitor markers CD133, EpCam (Figure 7e and f), and survivin (Supplementary Figure 7D). Malignant hepatocytes along the edge of this peritumoral stroma also expressed EpCam and 


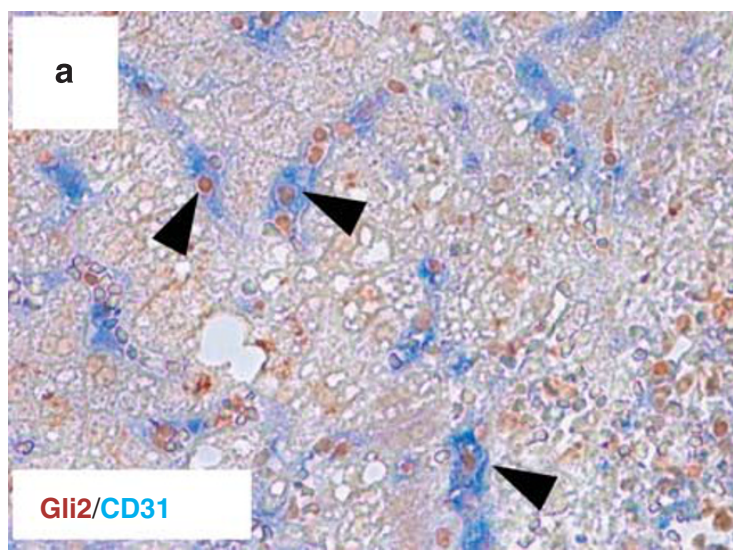

b
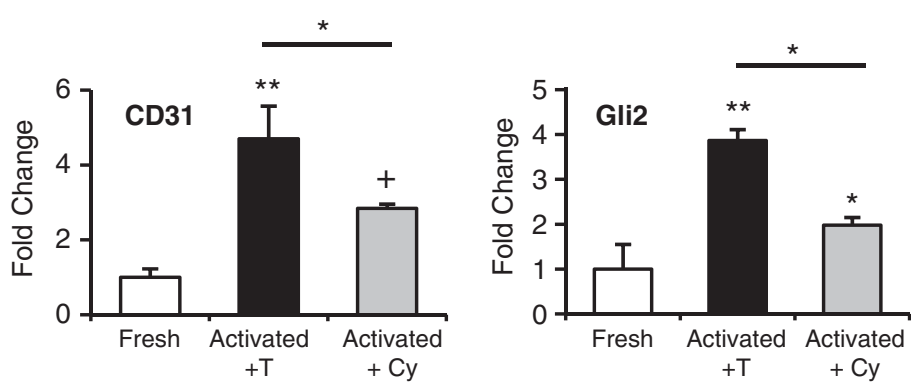

C

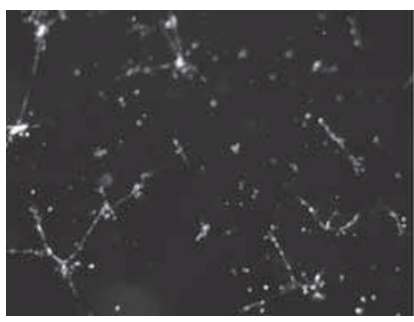

d Control media

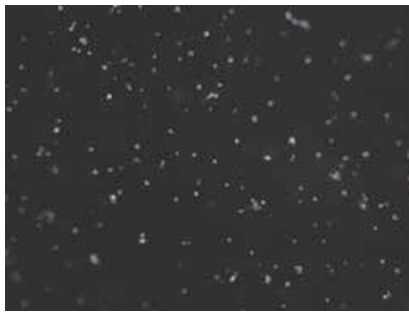

e

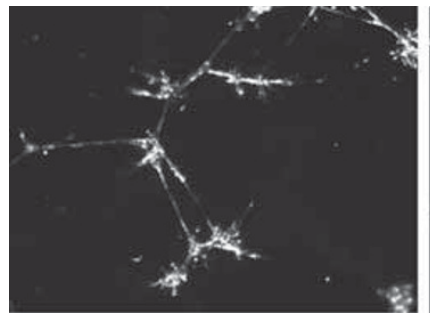

Shh $5 \mu \mathrm{g} / \mathrm{ml}$
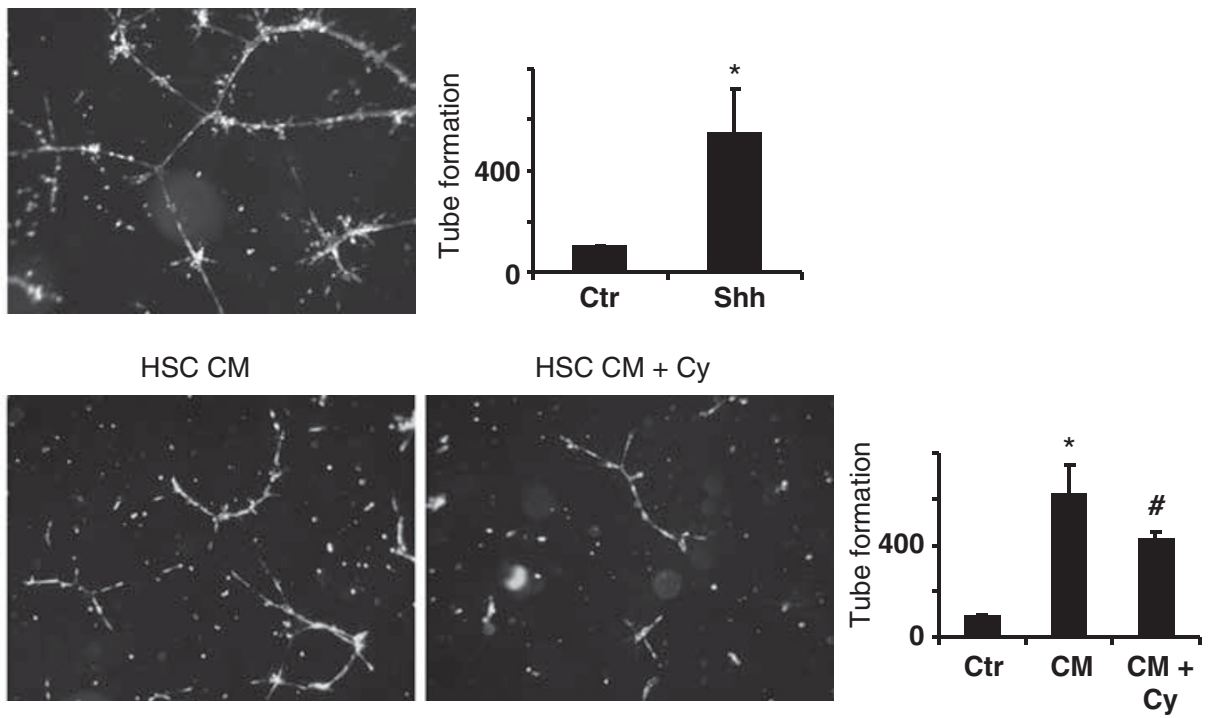

Figure 4 Activated endothelial cells are Hh responsive during chronic viral hepatitis and Hh pathway activation promotes vascular remodeling responses. (a) Immunohistochemistry was used to characterize the types of cells that accumulated in fibrotic septae. Representative photomicrograph from patient with stage F3/F4 chronic hepatitis $C$ is shown. Double immunohistochemistry for Gli2 (brown) and CD31 (blue). Final magnification $\times 600$. (b) Primary liver endothelial cells were isolated from a non-diseased liver; RNA was harvested from freshly isolated cells and from triplicate plates of cells that had been cultured to induce transition to an activated phenotype and then treated with either tomatidine (T) or cyclopamine (Cy) for an additional $48 \mathrm{~h}$. qRT-PCR analysis was done to determine effects on expression of CD31 (left) and Gli2 (right). Data were normalized to gene expression in the freshly isolated cells. Means \pm s.e.m. results are displayed. ${ }^{\star} P<0.05,{ }^{* *} P<0.005,{ }^{+} P<0.01$ vs fresh cells. (c, d) SECs were seeded into matrigel and treated with vehicle or Shh ligand $(5 \mu \mathrm{g} / \mathrm{ml})(\mathbf{c})$ and HSC condition media (CM) with or without Cy (d). (e) SECs were pre-treated with lentivirus containing Smo scramble (scr) shRNA or Smo shRNA. Effects on vascular tube formation were assessed in triplicate experiments. Representative results are displayed as arbitrary units. Means \pm s.e.m. are graphed. ${ }^{*} P<0.05$ vs control; ${ }^{\#} P<0.05$ vs condition medium alone.

survivin (Figure 7f, Supplementary Figure 7D), but not Krt7 (Figure 7d) or CD133 (Figure 7e). Hence, cells with features of tumor stem/initiating cells that accumulate in livers with chronic viral hepatitis are Hh responsive, and many produce
Shh. Such cells localize in ductular structures that are embedded in tumor-associated fibrous stroma that is enriched with cells that are Hh responsive (ie, Ptc and/or Gli2 positive), and that produce Ihh ligand. 

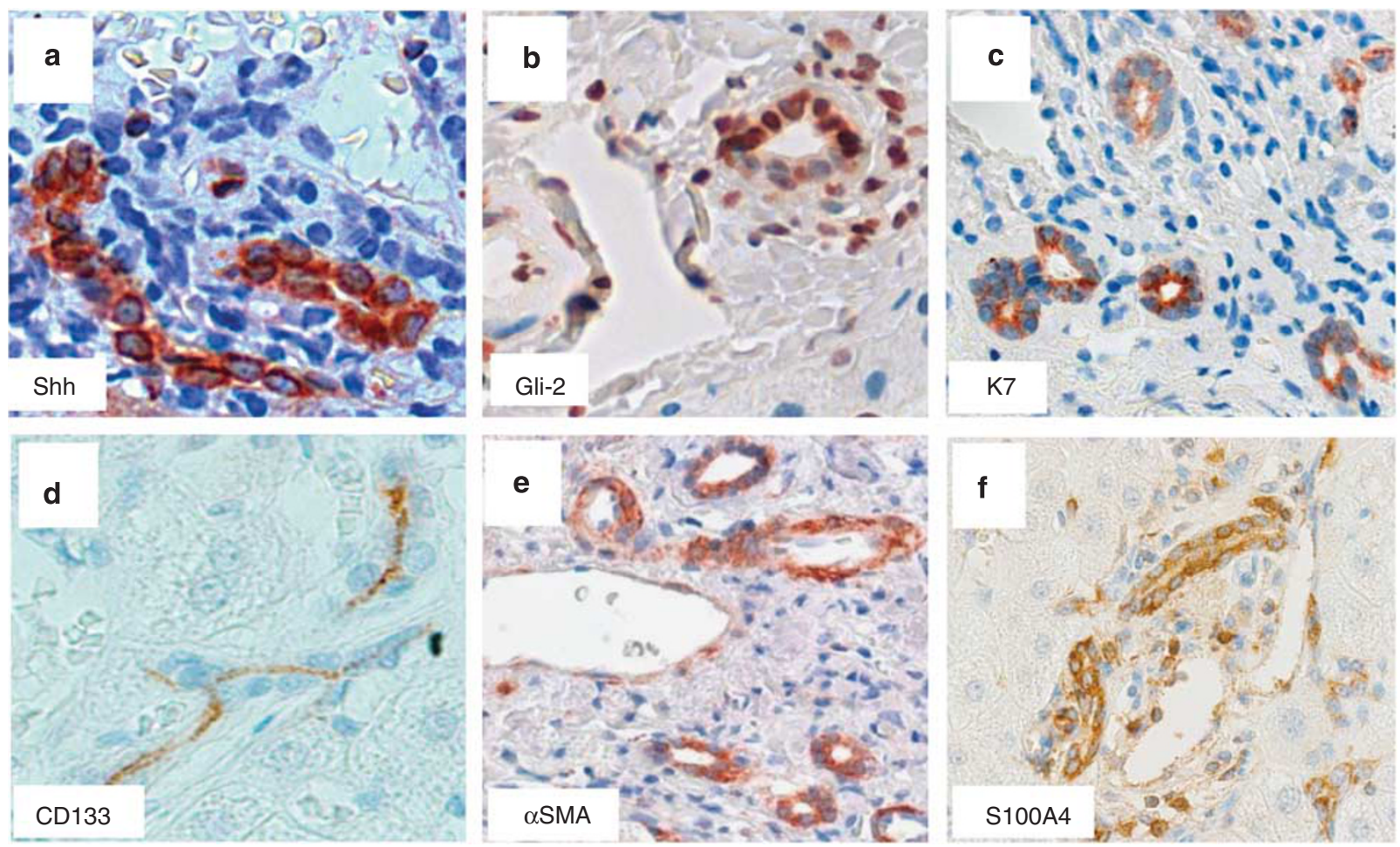

Figure 5 The ductular reaction that occurs during chronic viral hepatitis is enriched with Hh-responsive ductular cells that coexpress epithelial progenitor cell and mesenchymal cell markers. Immunohistochemistry was used to characterize the types of cells that accumulated in fibrotic septae. Representative photomicrographs from patients with stage F3/F4 chronic hepatitis C are shown. Monostaining for (a) Shh, (b) Gli2, (c) Krt 7, (d) CD133, (e) $\alpha$-SMA, and (f) S100A4. Final magnifications $\times 600$.

\section{DISCUSSION}

Chronic infection with HBV and HCV is a major cause of cirrhosis and liver cancer worldwide. ${ }^{2,3}$ Nevertheless, many individuals do not develop significant liver fibrosis or neoplasia, ${ }^{3,4}$ and although there is little argument that viral infection increases hepatocyte death, viral levels do not reliably predict important outcomes of liver injury. At present, the mechanisms that account for the variable consequences of chronic viral hepatitis are not fully understood.

We performed a cross-sectional analysis of liver biopsy samples from patients with chronic HBV or chronic HCV and with differing degrees of liver fibrosis. A subgroup of the patients had co-incident HCC. Our results suggest that interindividual differences in the degree of Hh pathway activation that occur during chronic viral infection may mediate some of the variability in viral hepatitis outcomes. We found that chronic infection with hepatitis viruses significantly increased hepatic expression of Hh ligands (Shh and Ihh) and target genes (Ptc and Gli2), and noted that individuals with more advanced stages of liver disease (ie, bridging fibrosis to cirrhosis) expressed higher levels of Shh, Ptc, and Gli2 than those with little or no fibrosis. In our study population, all of the HCCs occurred in livers with advanced fibrosis/cirrhosis; cirrhotic livers also tended to have the greatest net $\mathrm{Hh}$ pathway activity. Thus, higher levels of Hh pathway activity associated with both of the potentially fatal outcomes of chronic viral hepatitis (ie, cirrhosis and primary liver cancer).
The design of our study (retrospective, cross-sectional analysis) and nature of our study population (humans) prevented us from manipulating $\mathrm{Hh}$ signaling to test the potential cause-effect relationship between Hh pathway activity and outcomes of advanced virus-mediated liver disease in our study cohort. However, to overcome this limitation, we performed IHC to characterize the types of liver cells that produced and responded to Hh ligands in our patients, and then used cultured human liver cells to determine if (and how) manipulating Hh signaling altered the phenotypes of the relevant Hh-responsive cells.

IHC revealed that liver epithelial cells (ie, hepatocytes and ductular cells) were the major source of Shh ligands in patients with viral hepatitis, whereas stromal cells were the predominant producers of Ihh ligands in these subjects. Introducing HCV proteins into cultured liver epithelial cells induced significant expression of Shh mRNA but had no appreciable effect on Ihh expression. To our knowledge, these data provide the first evidence that hepatitis-viral infection of liver epithelial cells stimulates such cells to produce Shh ligand, a potent morphogen and viability factor for various stem/progenitor cells.

Immunostaining also demonstrated that the major liver cell populations that typically expand during cirrhosis (ie, myofibroblasts, activated SEC, and liver epithelial progenitors) are all enriched with cells that respond to Hh ligands. Treating cultures of primary human myofibroblastic HSCs 

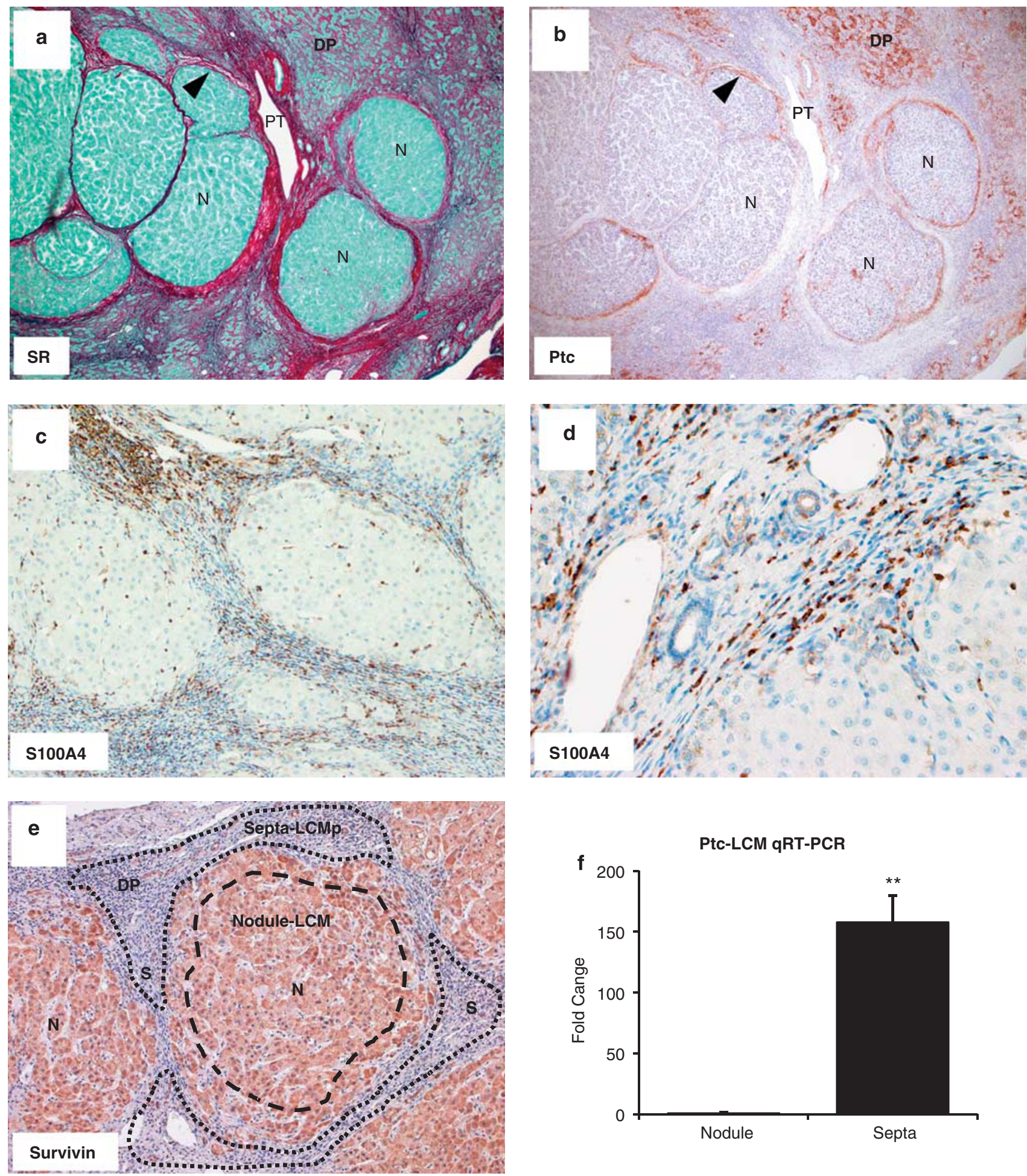

Figure 6 Peritumoral stromal tissue adjacent to HCC is enriched with Hh-responsive stromal cells and ductular cells that express markers of tumor stem/ progenitor cells. (a) Nodules of HCC (N) and adjacent peritumoral tissue in representative patient with chronic hepatitis $\mathrm{C}$ and HCC. Final magnification $\times$ 100. (b-e) Immunohistochemistry for Ptc (b), S100A4 (c, d), and survivin (e) in serial sections from this patient. Dotted lines delineate in $(\mathbf{e})$ margins of samples that were procured using laser capture dissection microscopy (LCM). Final magnifications $\times 100$ (b, c), $\times 400$ (d), and $\times 200$ (e). (f) qRT-PCR analysis of peritumoral fibrotic septae and nodules of malignant hepatocytes from three patients with chronic viral hepatitis and HCC. Data were normalized to Ptc gene expression in the tumor nodules. Means \pm s.e.m. are displayed. ${ }^{* \star P}<0.005$ vs tumor nodules. 

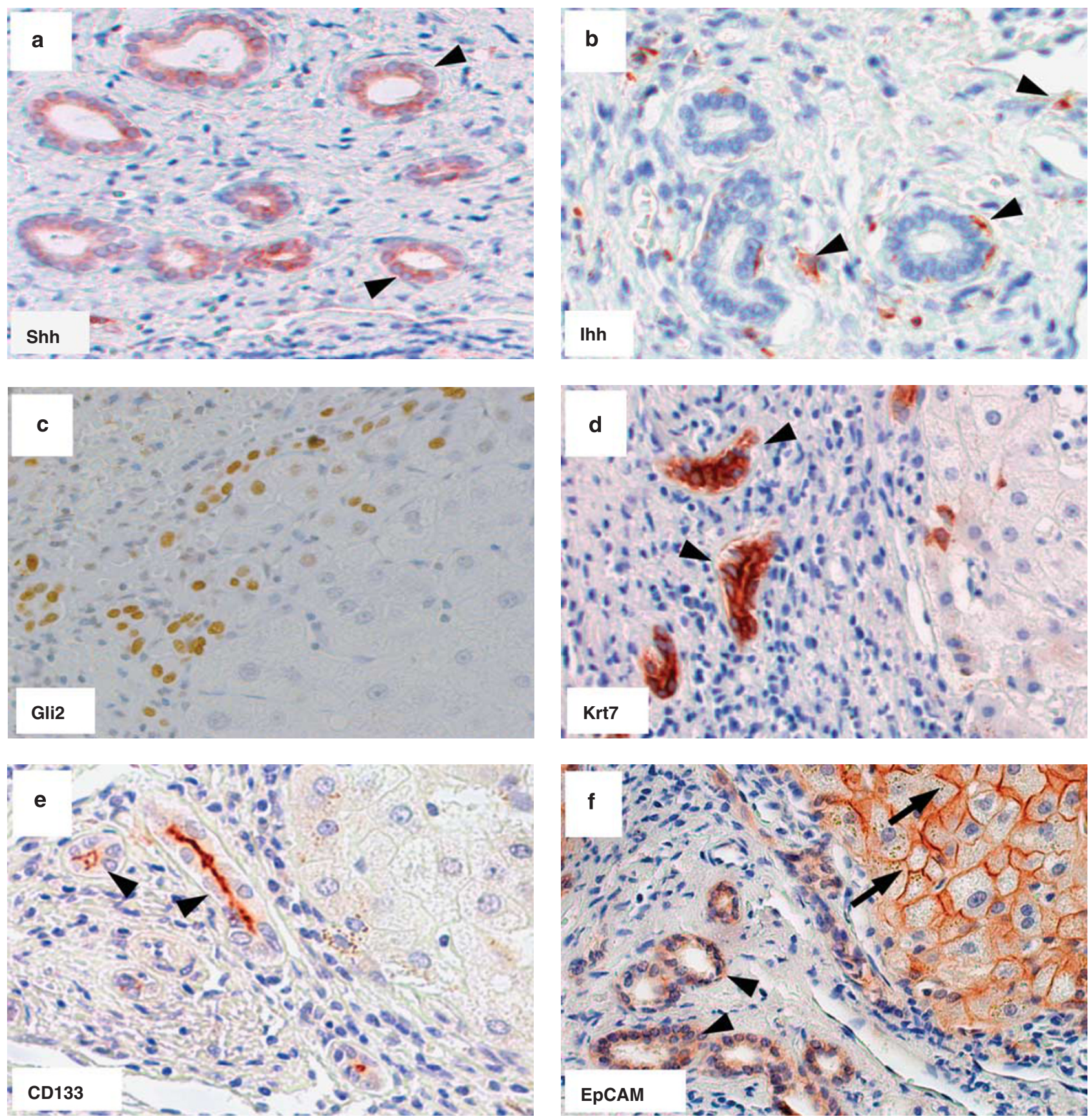

Figure 7 Peritumoral tissue adjacent to HCC is enriched with Hh-responsive cells. Immunohistochemistry was used to characterize the types of cells that accumulated in peritumoral fibrotic tissue. Representative photomicrographs are shown. Monostaining (arrowheads) for (a) Shh, (b) Ihh, (c) Gli2, (d) Krt7, (e) CD133, and (f) EpCam. Final magnifications $\times 600$ (a, b, d, e, f) or $\times 400$ (c).

with cyclopamine, a highly specific antagonist of Smo, inhibited Hh signaling and repressed expression of fibroblastassociated genes. Similarly, the $\mathrm{Hh}$ pathway was active in activated human liver SECs, and blocking Hh signaling with cyclopamine repressed the activated phenotype of such cells. Recombinant Shh also induced vascular tube formation in cultured human endothelial cells, and various strategies that inhibited Hh signaling prevented angiogenesis in that assay. We had previously demonstrated that Hh ligands promoted viability, growth, and EMT in cultures of immature ductular cells. $^{7-9}$ Together, these data indicate that Hh pathway activation is not merely an epiphenomenon that occurs during chronic viral hepatitis. Rather, it likely has an important causal role for the accumulation of myofibroblasts, activated endothelial cells, and hepatic epithelial progenitors during this disease. Hence, virally mediated increases in Hh ligand production activate $\mathrm{Hh}$ signaling in $\mathrm{Hh}$ target cells that reconstruct virally damaged livers, thereby influencing the 
levels of fibrosis, vascular remodeling, and progenitor accumulation that ensue.

Many types of adult cancers, including HCCs, exhibit aberrant activation of the Hh pathway. ${ }^{20,21,34}$ We were unable to demonstrate $\mathrm{Hh}$ ligand or target gene expression within nodules of HCCs in livers with chronic viral hepatitis, but noted striking enrichment of peritumoral stromal tissue with a spectrum of cells that produced and/or responded to $\mathrm{Hh}$ ligands, ranging from ductular-appearing cells to fibroblastic cells. Moreover, some of these peritumoral cells coexpressed markers of cancer stem/initiating cells and mesenchymal cells. Coexpression of epithelial and mesenchymal genes is a characteristic of cells that are undergoing EMT/MET. ${ }^{14,33}$ Hence, these findings suggest that 'epithelioid' nodules of HCCs were derived from subpopulations of malignantly transformed, Hh-responsive liver progenitors. Such tumor stem/initiating cells have relatively mesenchymal phenotypes, and seem to reside in peritumoral stroma until Hh signaling is repressed sufficiently to permit them to undergo MET, whereby they progressively acquire more hepatocytic features. Additional research is necessary to prove/disprove this concept. However, it is consistent with the known ability of Hh ligands to promote EMT, ${ }^{8,10}$ the fact that HCC cell lines display variable levels of $\mathrm{Hh}$ pathway activity and mesenchymal gene expression, ${ }^{35}$ evidence that viral factors induce EMT in liver cells, ${ }^{36,37}$ and findings of EMT-related gene expression in HCV-related HCC. ${ }^{15,38}$ Therefore, like cirrhosis, HCC may be a direct consequence of Hh-dependent remodeling of liver damage caused by chronic viral infection. If confirmed, such results would have important implications for patients with viral hepatitis because (1) interindividual differences in $\mathrm{Hh}$ responsiveness may underlie observed differences in the outcomes of chronic $\mathrm{HBV} / \mathrm{HCV}$ and (2) Hh inhibitors may help to prevent cirrhosis and HCC in patients with these diseases.

Supplementary Information accompanies the paper on the Laboratory Investigation website (http://www.laboratoryinvestigation.org)

\section{ACKNOWLEDGEMENTS}

We thank Professor Paulo RM Vargas, chief of Pathology at HUCAM/UFES, for sending the paraffin blocks. Huh-7 cells were a gift from Eckard Wimmer (Stony Brook University, NY, USA); HCV genotype 2a JFH-1 virus was a gift from P Goldschmidt-Clermont (University of Miami, FL, USA). This work was supported by RO1-DK077794 (to AMD); CAPES Fellowship from the Brazilian government (to TAP); CA136526, Mayo Clinic Pancreatic SPORE P50CA101701, and Mayo Clinic Center for Cell Signaling in Gastroenterology P30DK084567 (to MEF).

\section{DISCLOSURE/CONFLICT OF INTEREST}

The authors declare no conflict of interest.

1. Schuppan D, Afdhal NH. Liver cirrhosis. Lancet 2008;371:838-851.

2. Caldwell S, Park SH. The epidemiology of hepatocellular cancer: from the perspectives of public health problem to tumor biology. J Gastroenterol 2009;44(Suppl 19):96-101.

3. Fung J, Lai CL, Yuen MF. Hepatitis B and C virus-related carcinogenesis. Clin Microbiol Infect 2009;15:964-970.
4. Guidotti LG, Chisari FV. Immunobiology and pathogenesis of viral hepatitis. Annu Rev Pathol 2006;1:23-61.

5. Friedman SL. Mechanisms of hepatic fibrogenesis. Gastroenterology 2008;134:1655-1669.

6. Mishra L, Banker T, Murray J, et al. Liver stem cells and hepatocellular carcinoma. Hepatology 2009;49:318-329.

7. Jung $\mathrm{Y}$, Brown KD, Witek RP, et al. Accumulation of hedgehogresponsive progenitors parallels alcoholic liver disease severity in mice and humans. Gastroenterology 2008;134:1532-1543.

8. Omenetti $A$, Porrello $A$, Jung $Y$, et al. Hedgehog signaling regulates epithelial-mesenchymal transition during biliary fibrosis in rodents and humans. J Clin Invest 2008;118:3331-3342.

9. Syn WK, Jung $Y$, Omenetti $A$, et al. Hedgehog-mediated epithelial-tomesenchymal transition and fibrogenic repair in nonalcoholic fatty liver disease. Gastroenterology 2009;137:1478-1488.e8.

10. Omenetti A, Diehl AM. The adventures of sonic hedgehog in development and repair. II. Sonic hedgehog and liver development, inflammation, and cancer. Am J Physiol Gastrointest Liver Physiol 2008:294:G595-G598.

11. Sicklick JK, Li YX, Melhem A, et al. Hedgehog signaling maintains resident hepatic progenitors throughout life. Am J Physiol Gastrointest Liver Physiol 2006;290:G859-G870.

12. Syn WK, Witek RP, Curbishley SM, et al. Role for hedgehog pathway in regulating growth and function of invariant NKT cells. Eur J Immunol 2009;39:1879-1892.

13. Yang $L$, Wang $Y$, Mao $H$, et al. Sonic hedgehog is an autocrine viability factor for myofibroblastic hepatic stellate cells. J Hepatol 2008; 48:98-106.

14. Choi SS, Diehl AM. Epithelial-to-mesenchymal transitions in the liver. Hepatology 2009;50:2007-2013.

15. Jou J, Diehl AM. Epithelial-mesenchymal transitions and hepatocarcinogenesis. J Clin Invest 2010;120:1031-1034.

16. Omenetti A, Syn WK, Jung $Y$, et al. Repair-related activation of hedgehog signaling promotes cholangiocyte chemokine production. Hepatology 2009;50:518-527.

17. Asai J, Takenaka H, Kusano KF, li M, et al. Topical sonic hedgehog gene therapy accelerates wound healing in diabetes by enhancing endothelial progenitor cell-mediated microvascular remodeling. Circulation 2006;113:2413-2424.

18. Vokes SA, Yatskievych TA, Heimark RL, et al. Hedgehog signaling is essential for endothelial tube formation during vasculogenesis. Development 2004;131:4371-4380.

19. Witek RP, Yang L, Liu R, et al. Liver cell-derived microparticles activate hedgehog signaling and alter gene expression in hepatic endothelial cells. Gastroenterology 2009;136:320-330.e2.

20. Huang S, He J, Zhang $X$, et al. Activation of the hedgehog pathway in human hepatocellular carcinomas. Carcinogenesis 2006;27:1334-1340.

21. Sicklick JK, Li YX, Jayaraman A, et al. Dysregulation of the Hedgehog pathway in human hepatocarcinogenesis. Carcinogenesis 2006;27: 748-757.

22. Tada M, Kanai F, Tanaka $Y$, et al. Down-regulation of hedgehoginteracting protein through genetic and epigenetic alterations in human hepatocellular carcinoma. Clin Cancer Res 2008;14:3768-3776.

23. Kato $T$, Date $T$, Miyamoto $M$, et al. Efficient replication of the genotype $2 a$ hepatitis $C$ virus subgenomic replicon. Gastroenterology 2003;125:1808-1817.

24. Lindenbach BD, Evans MJ, Syder AJ, et al. Complete replication of hepatitis $C$ virus in cell culture. Science 2005;309:623-626.

25. Zhong J, Gastaminza P, Cheng G, et al. Robust hepatitis C virus infection in vitro. Proc Natl Acad Sci USA 2005;102:9294-9299.

26. Lopes N, Gregg D, Vasudevan S, et al. Thrombospondin 2 regulates cell proliferation induced by Rac1 redox-dependent signaling. Mol Cell Biol 2003;23:5401-5408.

27. Choi SS, Omenetti A, Witek RP, et al. Hedgehog pathway activation and epithelial-to-mesenchymal transitions during myofibroblastic transformation of rat hepatic cells in culture and cirrhosis. Am J Physiol Gastrointest Liver Physiol 2009;297:G1093-G1106.

28. DeLeve LD, Wang $X$, McCuskey MK, et al. Rat liver endothelial cells isolated by anti-CD31 immunomagnetic separation lack fenestrae and sieve plates. Am J Physiol Gastrointest Liver Physiol 2006;291: G1187-G1189.

29. Sicklick JK, Li YX, Choi SS, et al. Role for hedgehog signaling in hepatic stellate cell activation and viability. Lab Invest 2005;85:1368-1380. 
30. Semela $D$, Das $A$, Langer $D$, et al. Platelet-derived growth factor signaling through ephrin-b2 regulates hepatic vascular structure and function. Gastroenterology 2008;135:671-679.

31. Fernandez-Zapico ME, Gonzalez-Paz NC, Weiss E, et al. Ectopic expression of VAV1 reveals an unexpected role in pancreatic cancer tumorigenesis. Cancer Cell 2005;7:39-49.

32. Jiang J, Hui CC. Hedgehog signaling in development and cancer. Dev Cell 2008;15:801-812.

33. Kalluri $\mathrm{R}$, Weinberg RA. The basics of epithelial-mesenchymal transition. J Clin Invest 2009;119:1420-1428.

34. Katoh $Y$, Katoh $M$. Hedgehog target genes: mechanisms of carcinogenesis induced by aberrant hedgehog signaling activation. Curr Mol Med 2009;9:873-886.
35. Slany A, Haudek VJ, Zwickl H, et al. Cell characterization by proteome profiling applied to primary hepatocytes and hepatocyte cell lines Hep-G2 and Hep-3B. J Proteome Res 2010;9:6-21.

36. Battaglia S, Benzoubir N, Nobilet $\mathrm{S}$, et al. Liver cancer-derived hepatitis $C$ virus core proteins shift TGF-beta responses from tumor suppression to epithelial-mesenchymal transition. PLOS ONE 2009;4:e4355.

37. Yang $S Z$, Zhang LD, Zhang $Y$, et al. $H B x$ protein induces EMT through c-Src activation in SMMC-7721 hepatoma cell line. Biochem Biophys Res Commun 2009;382:555-560.

38. van Zijl F, Zulehner G, Petz $M$, et al. Epithelial-mesenchymal transition in hepatocellular carcinoma. Future Oncol 2009;5: 1169-1179. 